\title{
Tuberculous peritonitis: a review of thirty-five cases
}

\author{
S. VyRAVANATHAN \\ T.D.D. (Wales)
}

\author{
R. JEYARAJAH \\ M.R.C.P. (UK)
}

\begin{abstract}
Chest Clinic, Kandy, and Department of Medicine, Faculty of Medicine, University of Peradeniya, Sri Lanka
\end{abstract}

\begin{abstract}
Summary
The clinical course of 35 patients with tuberculous peritonitis notified in Kandy (Sri Lanka) over a 6-year period has been reviewed. The maximum incidence occurred in the age group 21 to 40 years and the female/male ratio was $3: 2$. Clinical features were non-specific and the main presenting features were fever $(68.5 \%)$, abdominal pain $(65.7 \%)$, abdominal distension $(54.2 \%)$, abdominal mass $(54.2 \%)$ and ascites $(45.7 \%)$. The symptoms were of insidious onset and $85 \%$ had symptoms for more than one month. Chest X-ray revealed abnormality suggestive of tuberculosis in $47 \%$. Intra-abdominal tuberculosis was suspected in $43 \%$ of 26 patients before laparotomy. Four of the 32 patients given anti-tuberculous drugs died. Overall mortality was $20 \%$.
\end{abstract}

\section{Introduction}

Tuberculosis continues to be a major health problem in Sri Lanka. The incidence of tuberculous peritonitis has been estimated to range from $0 \cdot 1 \%$ to $0.7 \%$ (Lougheed and Saporta, 1963; Olcott and Pacchione, 1933; Kahrs, 1952; Sochocky, 1967) of all reported cases of tuberculosis. A study has been made of the incidence, clinical features, diagnosis and treatment of this illness in Kandy.

\section{Materials and methods}

Of the 2716 cases of tuberculosis notified in Kandy during a 6-year period from March 1973, there were 43 patients with tuberculous peritonitis. Only 35 were used for this analysis as the data for the other 8 were not adequate. In 33, the diagnosis was made on histological examination of the peritoneum (laparotomy in 26, peritoneal biopsy with an Abrams' needle in one; at post-mortem in 6 ). Of the remaining 2 , one had a predominantly lymphocytic peritoneal fluid with histologically confirmed tuberculosis of cervical lymph node; the other had radiological evidence of generalized haematogenous tuberculosis and a faecal fistula. Mantoux test was done in 28 cases; chest X-ray in 34 ; $\mathrm{Hb}$ in 17; WBC in 15; and ESR in 15.
Results

The incidence of tuberculous peritonitis is $1.5 \%$ of all reported cases of tuberculosis in Kandy. The average age was $30 \cdot 1$ years with a range of 2 to 58 years, and 25 were aged $21-40$ years. There were $21(60 \%)$ females and $14(40 \%)$ males. The duration of symptoms varied from one week to one year. The major presenting symptoms were fever, abdominal pain, abdominal distension, anorexia and loss of

TABLE 1. Main presenting symptoms in 35 patients with tuberculous peritonitis

\begin{tabular}{llc}
\hline \multicolumn{1}{c}{ Symptoms } & $\begin{array}{c}\text { No. of } \\
\text { patients } \\
(\%)\end{array}$ & $\begin{array}{c}\text { No. with symptoms } \\
\text { of < one month's } \\
\text { duration }\end{array}$ \\
\hline Fever & $24(68 \cdot 5)$ & 21 \\
Abdominal pain & $23(65 \cdot 7)$ & 15 \\
Abdominal distension & $19(54 \cdot 2)$ & 14 \\
Anorexia & $14(40)$ & 14 \\
Loss of weight & $11(31 \cdot 4)$ & 11 \\
Cough & $2(5 \cdot 7)$ & \\
Vomiting & $3(8 \cdot 5)$ & \\
Amenorrhoea & $5(14 \cdot 2)$ & \\
Abdominal mass & $2(5 \cdot 7)$ & \\
Diarrhoea & $1(2 \cdot 8)$ & \\
\hline
\end{tabular}

weight (Table 1). Of the 2 patients who had a cough, one had had it for one week and the other for 3 months. In the former, chest X-ray showed bilateral upper zone opacities while in the latter there was bilateral pleural effusion. One of the 3 who complained of vomiting for 2 weeks had clinical and radiological evidence of small intestinal obstruction. The period of amenorrhoea ranged from 4 months to one year. Two of these patients had pelvic tuberculosis. The main physical findings were abdominal mass, abdominal distension, ascites, emaciation and generalized abdominal tenderness (Table 2). The Mantoux test was positive in 22 of the 28 patients in whom it was done $(78.5 \%) . \mathrm{Hb}$ was $<10 \mathrm{~g} / \mathrm{dl}$ in 6 ; WBC was $>10 \times 10^{9} / \mathrm{dl}$ in 9 with neutrophil leucocytosis; and ESR was $<25 \mathrm{~mm} / \mathrm{hr}$ 
in 4 patients. Chest X-ray showed abnormality in 18 (Table 3). Intra-abdominal tuberculosis was suspected in 12 of 26 patients who underwent laparotomy; 7 of these had radiological abnormalities suggestive of pulmonary tuberculosis. In the 6 who died, 5 had chest X-ray evidence of tuberculosis, but only 2 were suspected of having tuberculous peritonitis. In the sixth patient, chest X-ray could not be done but post-mortem examination revealed evidence of pulmonary tuberculosis. Laparotomy and post-mortem findings are given in Table 4. The adults were treated with the following regimen: streptomycin, $0.75 \mathrm{~g}$ i.m.; isoniazid, $300 \mathrm{mg}$; and PAS $10 \mathrm{~g}$ orally were given daily for a minimum period of 3 months; and streptomycin one $g$ and isoniazid $700 \mathrm{mg}$ twice weekly for a further 15 months. Prednisolone $20 \mathrm{mg}$ orally was also given daily for 2 weeks and gradually discontinued. Of the 32 treated patients, 4 died ( 3 within one week, the

TABLE 2. Main physical signs in 35 patients with tuberculous peritonitis

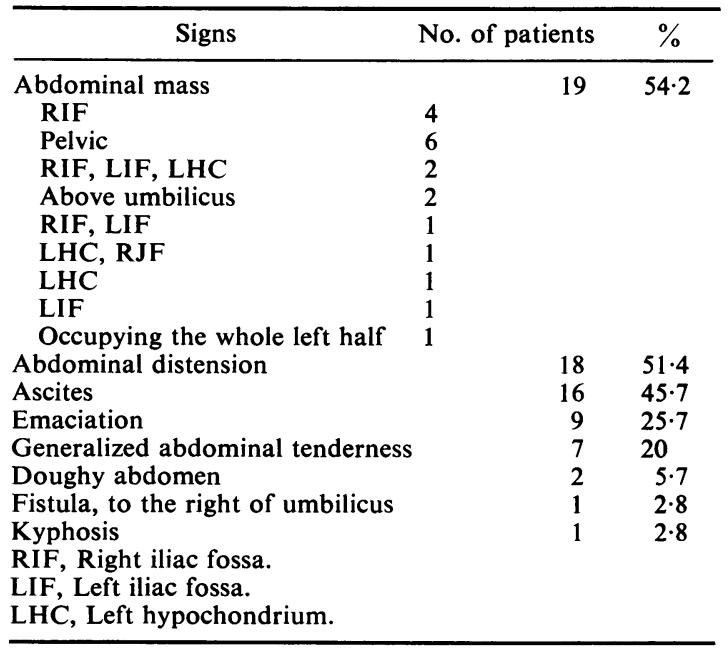

TABLE 3. Chest X-ray abnormalities in 18 patients with tuberculous peritonitis

\begin{tabular}{llc}
\hline \multicolumn{1}{c}{ Findings } & No. of patients \\
\hline $\begin{array}{c}\text { Pleural effusions } \\
\text { Effusions only (bilateral effusion in one) }\end{array}$ & 4 & 7 \\
$\quad \begin{array}{l}\text { Effusion with generalized haemato- } \\
\text { genous TB (bilateral effusion in one) }\end{array}$ & 2 & \\
$\begin{array}{l}\text { Effusion with chronic pulmonary TB } \\
\quad \text { (bilateral effusion in one) }\end{array}$ & 1 & \\
$\begin{array}{l}\text { Chronic pulmonary TB } \\
\text { Primary TB }\end{array}$ & & 2 \\
$\quad \begin{array}{l}\text { Right hilar lymphadenopathy } \\
\text { Right para-tracheal lymphadenopathy }\end{array}$ & 1 & 2 \\
Paravertebral abscess & 1 & \\
Non-specific-Fleishner's lines & & 1 \\
\hline
\end{tabular}

TABLE 4. Laparotomy and post-mortem findings in $32 \frac{8}{\mathbb{Q}}$ patients

\begin{tabular}{lcr}
\hline \multicolumn{1}{c}{ Findings } & No. of patients \\
\hline Free fluid in peritoneum & 30 & 32 \\
$\quad$ Straw-coloured & 1 & \\
Blood-stained & 1 & \\
$\quad$ Milky & & 21 \\
Miliary tubercles & 15 \\
Adhesions & 12 \\
Lymphadenopathy & 6 \\
Hyperplastic tuberculosis of caecum & 4 \\
Salpingitis & & \\
\hline
\end{tabular}

4th one month after commencement). The 28 who recovered have been followed-up for periods ranging from 3 months to 3 years and they are all doing well. Of the 3 who were not treated, one died of postoperative complications and the other 2 were not suspected of having tuberculosis.

\section{Discussion}

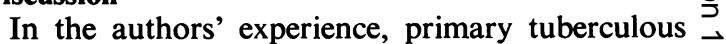
infection occurs mostly in the lungs. The spread os from this primary focus is by way of the blood $\mathbb{D}$ stream either directly to the peritoneum, or indirectly through abdominal lymph nodes or Fallopian tubes. This experience is supported by the following findings: presence of miliary tubercles over the $\vec{\varphi}$ peritoneum; tuberculous lymphadenitis; tuberculous salpingitis; and hyperplastic tuberculosis of the caecum (presumed to be of haematogenous origin by Armstrong, 1952). Auerbach (1950), described 2 forms of tuberculous peritonitis-the common caseous, and the less common generalized miliary. The latter apparently heals completely. However, in the present series, except for 4 of the 32, all had generalized miliary tuberculosis of the peritoneum. Sixty per cent. of the cases were female, which is similar to the incidence reported by Sochocky (1967). The higher incidence in women is attributed to the development of tuberculous peritonitis secondary to tuberculous salpingitis (Armstrong, 1952).

The onset in all the present cases was gradual and the symptoms were of more than one week's duration. Eighty-five per cent. had symptoms for $>$ one month before admission to hospital. At laparotomy and post-mortem (Table 4) the masses felt (Table 2) in the right iliac fossa (RIF) were found to be either thickened caecum and/or enlarged lymph nodes. Those found arising from the pelvis were due to pelvic peritonitis. Epigastric masses were rolled-up omentum; multiple, vague masses felt elsewhere in the abdomen were enlarged lymph nodes, and the huge mass occupying the left half consisted of adherent bowel, nodes and omentum. Six of the patients had thickening of the ileo-caecal region, suggesting ileo-caecal tuberculosis. Three of these 
had a solitary mass in the RIF and, in these, the pre-operative diagnosis was ileo-caecal tuberculosis or carcinoma of the colon.

There was evidence suggestive of tuberculosis in 16 of the 34 patients in whom chest X-ray was done. In other studies, radiological abnormalities of the chest were found in 55\% (Sochocky, 1967) and in $32 \%$ (Hughes, Carr and Geraci, 1960). An incidental radiological finding was the presence of Fleishner's lines in 2. These have been described in cases of peritonitis (Rabin, 1952). The Mantoux test was positive in $78.5 \%$ of the present cases which is close to the $76.5 \%$ in the series by Khoury, Payne and Harvey (1978). This test, like the Hb, WBC and the ESR, has not been of any help in establishing the diagnosis.

Difficulties in diagnosis arise when there is no evidence of extra-abdominal tuberculosis, especially when presented with an illness of short duration. In patients with ascites, the presence of straw-coloured fluid with predominance of lymphocytes on abdominal paracentesis is suggestive of tuberculous peritonitis. Confirmation can be achieved by histological demonstration of a tuberculous lesion on peritoneal biopsy. A Cope's needle was used for peritoneal biopsy by Levine (1968), who obtained a $100 \%$ success rate, while Singh, Bhargava and Jain (1969) had only $64 \%$ success. Each of these stressed that the method must only be used on patients with ascites. Culture and guinea-pig inoculation take considerable time before the results are available. Only 6 out of the 15 gave positive results in the series reported by Khoury et al., (1978) who concluded that laparotomy is a safe and sure method of obtaining an immediate visual diagnosis and peritoneal biopsy.

The outlook for this disease was gloomy before the advent of anti-tuberculous chemotherapy - the mortality being $49 \%$ (Dineen, Homan and Grafe, 1976). With the use of these drugs it is much brighter $-3.3 \%$ to $7 \%$ (Khoury et al., 1978; Dineen et al.,
1976). In the present series, 3 were not treated because one died of post-operative complication and 2 were diagnosed only at post-mortem. Of the 32 who were treated, 4 died $(12 \cdot 5 \%)$. It was felt that these 4 presented too late with an overwhelming infection.

\section{Acknowledgments}

We wish to thank the surgeons and gynaecologists of Kandy and Nawalapitiya for the operative findings and Professor G. E. Tennekoon for the post-mortem findings and histology reports.

\section{References}

Armstrong, B. (1952) In: Modern Practice in Tuberculosis, Vol 2. (Ed by Holmes, Sellors, T. \& Livingstone, J.L.). Butterworth and $\mathrm{Co}$, London.

Auerbach, O. (1950) Pleural, peritoneal and pericardial tuberculosis. American Review of Tuberculosis, 61, 845.

Dineen, P., Homan, W.P. \& Grafe, W.R.(1976) Tuberculous peritonitis: 43 years' experience in diagnosis and treatment. Annals of Surgery, 184, 717.

Hughes, H.J., CarR, D.T. \& GeraCI, J.E. (1960) Tuberculous peritonitis: a review of 34 cases with emphasis on the diagnostic aspects. Diseases of the Chest, 38, 42.

KaHRS, T. (1952) Tuberculous peritonitis: a follow-up study of 169 cases. Tubercle. London, 33, 132.

Khoury, G.A., Payne, C.R. \& Harvey, D.R. (1978) Tuberculosis of the peritoneal cavity. British Journal of Surgery, 65, 808.

LEVINE, H. (1968) Needle biopsy in diagnosis of tuberculous peritonitis. American Review of Respiratory Disease, 98, 519.

Lougheed, J.C. \& SAPorta, J.H. (1963) Treatment and current status of tuberculous peritonitis. American Surgeon, 29, 850.

OlcotT, C.T. \& Paccione, D. (1933) Tuberculous peritonitis. American Review of Tuberculosis, 28L, 27.

RABIN, C.B. (1952) X-ray Diagnosis of Chest Diseases. Williams \& Wilkins, Baltimore.

Singh, M.M., BhargaVA, A.N. \& JaIN, K.P. (1969) Tuberculous peritonitis: an evaluation of pathogenic mechanisms, diagnostic procedures and therapeutic measures. New England Journal of Medicine, 281, 1091.

SocHOCKY, S. (1967) Tuberculous peritonitis: a review of 100 cases. American Review of Respiratory Disease, 95, 398. 


\section{CORRIGENDUM}

\section{Postgraduate Medical Journal}

September 1980 Vol. 56 No. 659

Page 649 Table 1, right-hand column heading:

for 'No. with symptoms of < one month's duration'

read 'No. with symptoms of $>$ one month's duration' 